\title{
Composition, richness and floristic diversity along an elevational gradient in a semi-disturbed treeline ecotone, Bhaderwah, Jammu and Kashmir
}

\author{
Dinesh Singh \\ Institute of Mountain Environment, University of Jammu (J\&K), India \\ Anu Sharma \\ Institute of Mountain Environment, University of Jammu (J\&K), India \\ Neeraj Sharma* \\ Institute of Mountain Environment, University of Jammu (J\&K), India \\ *Corresponding author. E-mail: nirazsharma@gmail.com
}

\begin{abstract}
The paper deals with plant species richness - diversity relationship along the rising elevation in a semi-disturbed tree line ecotone in upper Bhaderwah, a part of lesser Himalaya 253 plant species distributed over 170 genera and 62 families were recorded during the intensive field surveys carried during May 2014 to September 2016. These included 11 trees, 29 shrubs and 213 herb species with 247 angiosperms and 6 gymnosperms. Family Asteraceae with 37 species dominated the study area. Maximum richness (179) was observed at sub-alpine zone (Site IV) while the trees $\left(H^{\prime}=1.74\right)$ and shrubs $\left(H^{\prime}=2.48\right)$ revealed maximum diversity at Site III and herbs at Site IV $\left(\mathrm{H}^{\prime}=4.60\right)$. The maximum evenness for trees, shrubs and herbs was exhibited by sites III $\left(\mathrm{J}^{\prime}=0.79\right), \mathrm{V}\left(\mathrm{J}^{\prime}=0.87\right)$ and $\mathrm{VI}$ $(\mathrm{J} '=0.94)$, respectively. The diversity and evenness showed an incremental rise along the rising elevation reaching maxima at the mid elevation with Site-IV being the species rich and Site-VII species deficit, which implies that sub-alpine and alpine forests need effective monitoring and conservation.
\end{abstract}

Keywords: Diversity, Elevation, Extreme environment, Monitoring and conservation, Richness, Sub-alpine, Vegetation composition

\section{INTRODUCTION}

The treeline ecotone, marked by the culmination of the forested zone is the most prominent ecological boundary governed by climatic factors in the high mountains (Holtmeier, 2003). In a broader sense, the alpine timberlines represent the upper limit of forest on a mountain (Wardle, 1974). A product of the stochastic nature of weather and resilience of plant life, it forms a complex transitional boundary in mountain environments harbouring unique vegetation types therein. Recent climate change has become one of the main drivers of shifts in the geographical distributions of plant species (Sykes, 2009; Parmesan et al., 2011; Pacifici et al., 2015; Kosanic et al., 2018). Changes in geographical distributions of vegetation at the local and regional scales can impact community composition, ecosystem function, and genetic diversity, which can make plants even more vulnerable to on-going environmental change (Butchart et al., 2010; Richardson et al., 2010; Vila and Ibanez, 2011). The dense and close forests above the tree line gradually give way to shrubs and/or meadows where trees become stunted and deformed by the severe cli- mate. Rather than moving upward in defined bands, shifting plant assemblages are influenced by local topography and soil moisture (Hall and Fagre, 2003). Other factors mentioned as significant causes include microtopography including tree islands themselves (Holtmeier and Broll, 1992), soil composition (Cairns, 1998), fire (Rochefort et al., 1994), winter injury (Cairns and Malanson, 1998), and aspects of tree reproduction including seed bank location and abundance (Cairns and Malanson, 1997).

The alpine tree line ecotone, despite a long history of observations and field investigations, remain relatively poorly understood in some respects when compared to other ecosystems. Proper understanding of forest structure is a pre-requisite to describe various ecological processes and model the functioning and dynamics of forests (Elourard et al., 1997). The various constituents of species diversity that govern the expression of traits include the number of species present (species richness), their relative abundance (species evenness), presence of the particular species (species composition), the interactions among species, and the spatial and temporal variation in these properties. Species diversity im- 
pacts the resilience and resistance of ecosystems to environmental changes as well as effecting the current functioning of ecosystems (Chapin et al., 2000). In an attempt to better understand the trend in species richness and diversity along the rising elevation, the field surveys were conducted along a tree line ridge in upper Bhaderwah, valley of Jammu and Kashmir. The complex physiographic and topographic complexity of the mountain ranges of upper Bhaderwah valley has resulted in extreme habitat and microclimatic heterogeneity influencing the current distribution of vegetation.

\section{MATERIALS AND METHODS}

Study area: Our study focused on topographically complex regions located along the southwestern mountain ranges of Upper Bhaderwah valley covered by floristically diverse old growth. The study area forms the south-western part of Chenab catchment namely Kailash Kund circuit divided into seven sites at a regular interval of $200 \mathrm{~m}$ each starting from Chattergalla $(2800 \mathrm{~m})$ to Kailash lake (4000 m) (Fig 1). The forests of the study area are mainly composed of Kharsu OakConifer mixed patches leading to alpine scrub, vast meadows and rocky out crops beyond. Besides forming a typical tree line ecotone with a good diversity of vegetation, the study area is exposed to moderate disturbances in the form of unscientific TMAP extraction, fuel wood burning, timber removal, overgrazing, browsing and trampling, pollution, etc. which is attributed to myriad of situations which last for the entire summers in these highlands (Figs 2-7)

Methodology: The detailed floristic investigations were carried out in the study area during May 2014 to September 2016. $100 \mathrm{~m}^{2}$ quadrats, twenty each were laid at all seven sites in an elevational range of $1200 \mathrm{~m}(2800 \mathrm{~m}$ to $4000 \mathrm{~m})$. In each $10 \mathrm{~m} \times 10 \mathrm{~m}$ quadrat, the smaller quadrats of $25 \mathrm{~m}^{2}$ and $1 \mathrm{~m}^{2}$ each were laid for shrubs and herbs, respectively. Plants were collected (for herbarium) and photographed on the spot for identification. Proper care was taken to avoid any loss to the plants and habitat bearing them. The plant samples were collected in newspaper and compressed with the help of herbarium press, dried and finally mounted on herbarium sheets. The voucher specimens have been kept in the herbarium of Institute of Mountain Environment. Plant identification was mostly done on the field with the help of field guide viz. Flowers of the Himalaya (Polunin and Stainton 1984) besides consulting taxonomic experts of the region. Three vegetation layers, i.e. tree, shrub and herb were analyzed for species richness, diversity and dominance. Species richness was simply considered as the number of species per unit area (Whittaker, 1972). The richness was obtained by using the formula
$\mathrm{SR}=\mathrm{S}-1 / \mathrm{ln}(\mathrm{N})$ given by Margalef (1968) and $\mathrm{SR}=\mathrm{s} / \sqrt{ } \mathrm{n}$ (Menhinick, 1964) (where, $\mathrm{S}=$ number of species and $\mathrm{N}=$ Total number of individuals (of all species in case of Menhinick's index). The Shannon-Weiner diversity Index (Shannon and Wiener, 1963) was calculated by using formula $H^{\prime}=-\sum n i / n$ log2 ni/n (where ni is the IVI of a species and $n$ the sum total IVI values of all species in a forest type) while the evenness (equability) was obtained using the formula $\mathrm{E} 1=\mathrm{H}^{\prime} / \operatorname{In}$ (s) (Pielou, 1966). Dominance was calculated using Simpson's index (Simpson, 1949) using D: I-Cd, Where $\mathrm{Cd}=$ Simpson's concentration of dominance $=\left(\sum \mathrm{ni} / \mathrm{n}\right)^{2}$.

\section{RESULTS}

Floristic composition: The study area forms a typical tree line ecotone with the dominance of Kharsu Oak climax community (along the ridges) interspersed with Fir-Spruce-Birch-Bluepine mixture along the northern aspect. The Kharsu Oak community at sub-alpine zone is taken over by the Rhododendron-Juniper scrub and Krummholtz (stunted deformed vegetation) around $3800 \mathrm{~m}$ and the rocky outcrops beyond. These alpine landscapes are rich repositories of medicinal and aromatic plants presently under severe threats from different sources. The threatened medicinal plants found in the study area include Aconitum heterophyllum, A. violaceum, Arnebia benthamii, Artemisia maritima, Bergenia stracheyi, Betula utilis, Cypripedium cordigerum, C himalaicum, DactyIorhiza hatagirea, Fritilaria roylei, Hypericum perforatum, Inula aculeata, Jurinea macrocephala, Meconopsis aculeata, Pickrorhiza kurroa, Rhodiola heterodonta, Rhododendron anthopogon, $R$. companulatum, Sinopodophyllum hexandrum and Taxus wallichiana.

During the extensive field surveys along the tree line of upper Bhaderwah, we recorded 253 species of plants (11 trees, 29 shrubs and 213 herbs) contained in 170 genera and 62 families (Appendix 1, Table 1). Of these 247 are angiosperms (including 12 species of orchids) and 6
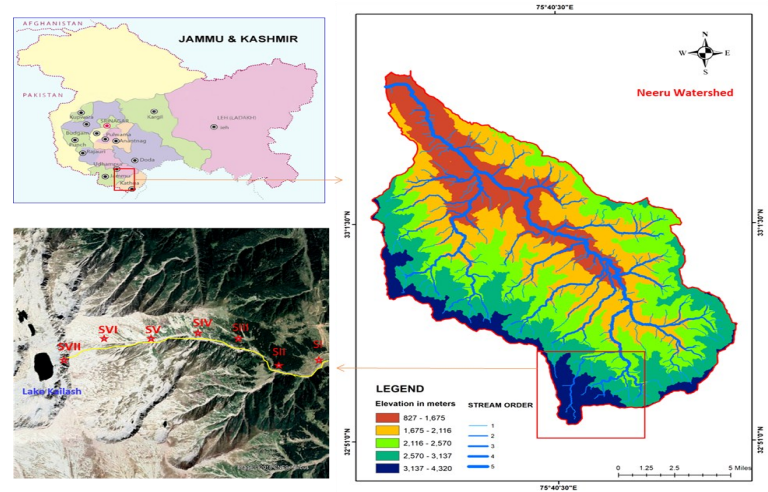

Fig. 1. Map of the study area showing different sites along the rising elevation. 
Appendix-1. Family wise species distribution along an elevational gradient $(2800-4000 \mathrm{~m})$ in a treeline ecotone of Bhaderwah.

\begin{tabular}{|c|c|c|c|c|c|c|c|c|}
\hline FAMILY / SPECIES & HABIT & SI & SII & SIII & SIV & sv & SVI & SVII \\
\hline Acanthaceae & & & & & & & & \\
\hline $\begin{array}{l}\text { Strobilanthes urticifolia Wall. } \\
\text { Aceraceae }\end{array}$ & Shrub & + & + & + & - & - & - & - \\
\hline $\begin{array}{l}\text { Acer acuminatum Wall. } \\
\text { Adoxaceae }\end{array}$ & Tree & + & + & + & - & - & - & - \\
\hline Viburnum cotinifolium D. Don & Shrub & - & + & + & - & - & - & - \\
\hline $\begin{array}{l}\text { Viburnum grandiflorum Wall. } \\
\text { Amaranthaceae }\end{array}$ & Shrub & + & + & + & - & + & - & - \\
\hline $\begin{array}{l}\text { Achyranthes bidentata Blume. } \\
\text { Anacardiaceae }\end{array}$ & Herb & + & + & - & - & - & - & - \\
\hline Toxicodendron succedaneum (L.) Kuntze & Tree & + & + & + & + & - & - & - \\
\hline Apiaceae & & & & & & & & \\
\hline Bupleurum longicaule Wall. ex DC. & Herb & + & + & + & + & + & + & - \\
\hline Heracleum candicans Wall. ex DC. & Herb & & + & + & + & + & - & - \\
\hline $\begin{array}{l}\text { Hymenidium stellatum (D. Don) M.G Pim- } \\
\text { nov \& E.V Kljuykov. }\end{array}$ & Herb & - & - & - & - & + & + & + \\
\hline $\begin{array}{l}\text { Pleurospermum brunonis Benth. ex } \\
\text { C.B.Clarke. }\end{array}$ & Herb & - & - & - & + & + & + & + \\
\hline Selinum filicifolium (Edgew.) Nasir. & Herb & + & + & + & + & + & - & - \\
\hline $\begin{array}{l}\text { Selinum wallichianum (DC.) Raizada \& } \\
\text { Saxena. } \\
\text { Araceae }\end{array}$ & Herb & - & + & + & + & - & - & - \\
\hline Arisaema flavum (Forssk.) Schott. & Herb & + & + & + & + & + & - & - \\
\hline Arisaema jacquemontii Blume. & Herb & + & + & + & + & - & - & - \\
\hline Arisaema propinquum Schott. & Herb & + & + & + & + & - & - & - \\
\hline $\begin{array}{l}\text { Arisaema tortuosum Wall. } \\
\text { Araliaceae }\end{array}$ & Herb & - & + & + & - & - & - & - \\
\hline $\begin{array}{l}\text { Hedera nepalensis K.Koch. } \\
\text { Asclepiadaceae }\end{array}$ & Herb & + & + & - & - & - & - & - \\
\hline $\begin{array}{l}\text { Vincetoxicum hirundinaria Medik. } \\
\text { Asparagaceae }\end{array}$ & Herb & + & - & + & + & - & - & - \\
\hline Maianthemum purpureum Wall. & Herb & - & + & - & - & + & - & - \\
\hline $\begin{array}{l}\text { Polygonatum verticillatum Mill. } \\
\text { Asteraceae }\end{array}$ & Herb & + & - & + & + & + & - & - \\
\hline Achillea millefolium $\mathrm{L}$. & Herb & + & + & + & + & + & - & - \\
\hline Anaphalis margaritacea L. & Herb & + & + & + & + & + & - & - \\
\hline Anaphalis nepalensis Hand. -Mazz. & Herb & & + & + & + & + & + & + \\
\hline Anaphalis triplinervis Sims ex C.B.Clarke. & Herb & + & + & + & + & + & - & - \\
\hline Arctium lappa L. & Herb & - & - & + & + & + & - & - \\
\hline Artemisia maritima L. & Herb & + & + & + & + & + & + & - \\
\hline Artemisia roxburghiana Wall.ex Besser. & Herb & + & + & + & - & + & - & - \\
\hline Aster flaccidus Bunge. & Herb & - & - & + & + & + & + & + \\
\hline Aster himalaicus Ganesh. & Herb & - & - & + & + & + & + & + \\
\hline Aster thomsonii C.B. Clarke & Herb & & + & + & + & + & - & - \\
\hline Carduus edelbergii Rech.f. & Herb & + & - & + & - & - & - & - \\
\hline Cicerbita macrorhiza (Royle) & Herb & - & - & + & + & + & - & + \\
\hline Cirsium falconeri (Hook.f.) Petr. & Herb & + & + & + & + & - & - & - \\
\hline Cirsium wallichii (L.) Scop. & Herb & + & + & - & - & - & - & - \\
\hline $\begin{array}{l}\text { Cremanthodium arnicoides (DC.ex } \\
\text { Royle) }\end{array}$ & Herb & - & + & + & - & - & - & + \\
\hline Cremanthodium ellisii (Hook.f.) Kitam. & Herb & - & + & + & + & - & - & + \\
\hline Dolomiaea macrocephala DC. & Herb & - & - & + & + & - & + & + \\
\hline Doronicum kamaonense DC. & Herb & + & + & + & + & - & - & - \\
\hline Erigeron multiradiatus Lindl. & Herb & - & + & + & + & + & - & - \\
\hline $\begin{array}{l}\text { Gnaphalium stewartii C.B.Clarke ex } \\
\text { Hook.f. }\end{array}$ & Herb & + & + & - & + & + & + & - \\
\hline Inula racemosa Hook.f. & Herb & - & + & + & - & - & + & + \\
\hline Inula royleana DC. & Herb & + & & + & + & + & - & + \\
\hline
\end{tabular}


Singh, D. et al. / J. Appl. \& Nat. Sci. 11(1): 23 - 34 (2019)

\begin{tabular}{|c|c|c|c|c|c|c|c|c|}
\hline Lactuca brunoniana (DC) Wall. ex C.B.Clarke. & Herb & - & + & - & + & + & - & - \\
\hline Lactuca lessertiana DC. & Herb & - & - & + & + & + & + & - \\
\hline Leontopodium himalayanum DC. & Herb & - & - & + & + & + & + & + \\
\hline Ligularia amplexicaulis DC. & Herb & - & - & + & + & + & + & - \\
\hline Ligularia fischeri (Ledeb.) Turcz. & Herb & + & + & + & - & + & + & - \\
\hline Ligularia Jacquemontiana Col. & Herb & - & + & - & - & - & + & + \\
\hline Onopordum acanthium L. & Shrub & + & + & - & - & - & - & - \\
\hline Saussurea atkinsonii C.B.Clarke & Herb & - & - & + & + & + & + & - \\
\hline Saussurea roylei C.B.Clarke & Herb & - & + & + & & + & + & - \\
\hline Saussurea taraxacifolia Wall. ex DC. & Herb & - & - & + & + & + & + & + \\
\hline Senecio laetus DC. & Herb & + & - & - & - & - & - & - \\
\hline Senecio raphanifolius Wall. & Herb & - & + & + & + & + & - & - \\
\hline Solidago virgaurea L. & Herb & - & + & - & + & + & - & - \\
\hline Tanacetum dolichophyllum Kitam. & Herb & - & - & + & + & + & + & + \\
\hline Taraxacum officinale Wigg. & Herb & + & + & + & + & + & - & + \\
\hline \multicolumn{9}{|l|}{ Balsaminaceae } \\
\hline Impatiens edgeworthii Hook.f. & Herb & + & + & + & - & - & - & - \\
\hline Impatiens glandulifera Royle. & Herb & - & + & + & + & - & - & - \\
\hline Impatiens sulcata Wall. & Herb & + & & + & + & + & + & - \\
\hline \multicolumn{9}{|l|}{ Berberidaceae } \\
\hline Berberis lycium Royle. & Shrub & + & + & - & - & - & - & - \\
\hline Sinopodophyllum hexandrum Royle. & Herb & + & + & + & + & + & - & - \\
\hline \multicolumn{9}{|l|}{ Betulaceae } \\
\hline \multicolumn{9}{|l|}{ Boraginaceae } \\
\hline Cynoglossum wallichii Wall. ex Benth & Herb & - & + & + & + & - & - & - \\
\hline Hackelia uncinata (Benth.) C.E.C.Fisch. & Herb & + & + & + & + & + & - & - \\
\hline Lindelofia longiflora (Benth.) & Herb & - & + & + & - & - & + & - \\
\hline \multicolumn{9}{|l|}{ Brassicaceae } \\
\hline Cardamine macrophylla Willd. & Herb & - & + & + & + & + & - & - \\
\hline Erysimum melicentae Dunn. & Herb & + & + & + & - & - & - & - \\
\hline Thlaspi montanum DC. & Herb & - & - & + & + & + & - & - \\
\hline \multicolumn{9}{|l|}{ Campanulaceae } \\
\hline Campanula aristata Wall. & Herb & - & - & + & + & - & - & - \\
\hline Campanula latifolia Lindl. & Herb & - & + & + & + & - & - & - \\
\hline Cyananthus lobatus Wall. ex Benth. & Herb & - & - & + & + & - & + & + \\
\hline Codonopsis ovata Benth. & Herb & - & + & + & - & + & - & - \\
\hline \multicolumn{9}{|l|}{ Caprifoliaceae } \\
\hline Dipsacus inermis Wall. & Herb & + & - & + & + & - & - & - \\
\hline Lonicera obovata Royle ex Hook. f. & Shrub & - & + & + & + & - & - & - \\
\hline Lonicera quinquelocularis L. & Shrub & + & + & - & - & - & - & - \\
\hline Morina longifolia Wall. & Herb & + & + & + & + & + & - & - \\
\hline Valeriana hardwickii Wall. & Herb & - & - & + & + & - & + & - \\
\hline Valeriana pyrolaefolia Decne. & Herb & - & + & + & + & - & - & - \\
\hline \multicolumn{9}{|l|}{ Caryophyllaceae } \\
\hline Cerastium cerastoides (L.) Britton. & Herb & - & - & - & + & + & - & + \\
\hline Gypsophila cerastioides D.Don. & Herb & - & + & + & + & + & - & - \\
\hline
\end{tabular}


Singh, D. et al. / J. Appl. \& Nat. Sci. 11(1): 23 - 34 (2019)

Myosoton aquaticum (L.) Moench

Silene cashmeriana (Benth. Ex Royle)

Silene gonosperma (Rupr.)

Silene vulgaris $\mathrm{L}$.

Stellaria decumbens Wight ex Edgew.

Stellaria himalayensis Wight.

\section{Celastraceae}

Parnassia nubicola Wall.

\section{Colchicaceae}

Colchicum luteum Baker.

\section{Crassulaceae}

Rhodiola fastigiata (Hook. $f$ and Thomson)

Rhodiola heterodonta (Hook. $\mathrm{f}$ and Thomson)

Rhodiola himalensis (D. Don) S.H. Fu.

Sedum ewersii Ledeb.

Sedum multicaule Wall.ex Lindl.

Sedum oreades (Decne.) Raym.-Hamet

\section{Cupressaceae}

Juniperus communis L.

Juniperus recurva Buch. -Ham. ex D.Don.

\section{Cyperaceae}

Carex nivalis. L.

\section{Ericaceae}

Cassiope fastigiata (Wall.) D.Don

Gaultheria trichophylla Royle.

Rhododendron anthopogon D.Don.

Rhododendron campanulatum D. Don.

\section{Euphorbiaceae}

Euphorbia cornigera Boiss.

Euphorbia wallichii Col.

\section{Fabaceae}

Campylotropis griffithii (Schindl.)

Desmodium elegans DC.

Indigofera hebepetala Benth. ex Baker.

Indigofera heterantha Wall.ex Brandis.

Lathyrus laevigatus (Waldst. and Kit.)

Trifolium pratense $\mathrm{L}$.

Trifolium repens $\mathrm{L}$.

\section{Fagaceae}

Quercus semecarpifolia D,Don.

Fumariaceae

Corydalis cashmeriana Royle.

Corydalis cornuta Royle.

Corydalis govaniana Wall

\section{Gentianaceae}

Gentiana carinata (D.Don) Griseb.

Gentiana venusta (G.Don) Wall. ex Griseb.

Gentianella moorcroftiana (Wall. ex G. Don) Airy Shaw

Jaeschkea oligosperma Knobl.

Swertia ciliata (D. Don ex G. Don) B.L. Burtt.

Swertia petiolata D.Don.

\begin{tabular}{|c|c|c|c|c|c|c|c|}
\hline Herb & + & + & - & - & - & - & - \\
\hline Herb & - & - & + & + & + & - & + \\
\hline Herb & - & - & + & + & - & + & + \\
\hline Herb & - & + & + & + & - & - & - \\
\hline Herb & - & - & - & + & + & - & + \\
\hline Herb & - & - & + & + & + & + & - \\
\hline Herb & + & - & - & - & - & - & - \\
\hline Herb & + & - & - & - & - & - & - \\
\hline Herb & - & - & - & - & + & + & + \\
\hline Herb & - & - & + & + & + & - & - \\
\hline Herb & - & - & + & + & + & + & - \\
\hline Herb & + & + & - & + & + & + & - \\
\hline Herb & - & + & + & + & - & - & - \\
\hline Herb & - & - & + & + & - & + & + \\
\hline Shrub & + & + & + & + & + & + & - \\
\hline Shrub & - & - & + & + & + & + & - \\
\hline Herb & - & - & - & - & - & + & + \\
\hline Shrub & - & - & - & + & + & + & + \\
\hline Shrub & - & - & + & + & + & + & + \\
\hline Shrub & - & - & & + & + & + & - \\
\hline Shrub & - & + & + & + & + & + & - \\
\hline Herb & + & + & + & + & + & - & - \\
\hline Herb & + & + & + & + & + & - & - \\
\hline Herb & - & - & + & + & - & + & - \\
\hline Shrub & + & + & - & - & - & - & - \\
\hline Shrub & - & + & + & + & - & - & - \\
\hline Shrub & - & + & - & + & + & - & - \\
\hline Herb & + & + & + & - & - & - & - \\
\hline Herb & + & + & - & - & - & - & - \\
\hline Herb & + & + & + & + & + & - & - \\
\hline Tree & + & + & + & + & - & - & - \\
\hline Herb & - & - & - & + & - & - & + \\
\hline Herb & - & + & + & - & + & - & - \\
\hline Herb & + & - & + & + & - & + & - \\
\hline Herb & - & - & + & + & + & + & - \\
\hline Herb & - & + & + & + & - & - & + \\
\hline Herb & + & + & + & + & + & + & + \\
\hline Herb & + & + & + & + & - & - & + \\
\hline Herb & + & + & + & + & + & - & - \\
\hline Herb & - & + & + & + & + & + & + \\
\hline
\end{tabular}




\begin{tabular}{|c|c|c|c|c|c|c|c|c|}
\hline \multicolumn{9}{|l|}{ Geraniaceae } \\
\hline Geranium nepalense Sweet. & Herb & + & + & + & - & - & - & - \\
\hline Geranium wallichianum D.Don ex Sweet & Herb & + & + & + & + & + & - & - \\
\hline \multicolumn{9}{|l|}{ Grossulariaceae } \\
\hline Ribes orientale Desf. & Shrub & + & + & - & + & - & - & - \\
\hline \multicolumn{9}{|l|}{ Hypericaceae } \\
\hline Hypericum elodeoides Wall. & Herb & + & + & + & + & - & - & - \\
\hline Hypericum perforatum L. & Herb & + & + & + & - & - & - & - \\
\hline \multicolumn{9}{|l|}{ Iridaceae } \\
\hline Iris hookeriana L. & Herb & - & - & - & + & + & - & - \\
\hline \multicolumn{9}{|l|}{ Juncaceae } \\
\hline Juncus himalensis Klotzsch. & Herb & - & + & - & + & - & - & + \\
\hline \multicolumn{9}{|l|}{ Lamiaceae } \\
\hline Clinopodium vulgare L. & Herb & + & + & + & - & + & - & - \\
\hline Lamium album $\mathrm{L}$. & Herb & + & + & - & + & + & + & + \\
\hline Leonurus cardiaca L. & Herb & + & - & + & + & - & - & - \\
\hline Mentha longifolia (L.) Huds. subsp. himalayensis (Briq.) Briq. & Herb & + & + & + & - & - & - & - \\
\hline Nepeta connata Royle ex Benth. & Herb & - & + & - & + & + & & + \\
\hline Nepeta laevigata (D.Don)Hand.-Mazz & Herb & - & + & + & + & - & + & - \\
\hline Nepeta leucophylla Benth. & Herb & + & + & + & - & + & - & - \\
\hline Origanum vulgare L. & Herb & - & + & + & + & - & - & - \\
\hline Phlomis bracteosa Royle ex Benth. & Herb & - & + & + & - & + & + & + \\
\hline Phlomis spectablis Falc. ex Benth. & Herb & + & - & + & + & + & - & - \\
\hline Prunella vulgaris $\mathrm{L}$. & Herb & + & + & + & - & + & - & - \\
\hline Salvia hians Royle ex Benth. & Herb & + & + & + & + & + & - & - \\
\hline Salvia nubicola Wall. & Herb & + & - & + & + & + & - & + \\
\hline Stachys emodi Wall. & Herb & + & + & + & - & + & + & - \\
\hline Stachys melissifolia Benth. & Herb & + & - & + & + & + & - & - \\
\hline Thymus linearis Benth. & Shrub & + & + & + & + & + & - & - \\
\hline \multicolumn{9}{|l|}{ Leguminosae } \\
\hline Lotus corniculatus L. & Herb & - & - & + & + & + & - & - \\
\hline Oxytropis lapponica (Wahlenb.) Gay. & Herb & - & - & + & + & + & + & - \\
\hline \multicolumn{9}{|l|}{ Liliaceae } \\
\hline Fritillaria roylei Hook. & Herb & - & + & + & + & + & - & + \\
\hline \multicolumn{9}{|l|}{ Oleaceae } \\
\hline Syringa emodi Wall. & Shrub & - & + & + & + & - & - & - \\
\hline \multicolumn{9}{|l|}{ Onagraceae } \\
\hline Epilobium royleanum Hausskn. & Herb & - & + & + & + & + & - & + \\
\hline \multicolumn{9}{|l|}{ Orchidaceae } \\
\hline Androcorys josephi (Rchb.f.) & Herb & - & - & + & - & - & - & - \\
\hline Calanthe tricarinata Lindl. & Herb & + & + & - & - & - & - & - \\
\hline Cypripedium cordigerum D. Don & Herb & + & - & - & - & - & - & \\
\hline Cypripedium himalaicum Rolfe. & Herb & - & - & + & + & - & - & - \\
\hline Dactylorhiza hatagirea D.Don & Herb & + & - & + & + & - & - & - \\
\hline Epipactis helleborine L. & Herb & - & - & + & + & - & - & - \\
\hline Gastrodia falconeri D.L.Jones and M.A.Clem. & Herb & + & - & - & - & - & - & - \\
\hline Goodyera repens (L.) R.Br. & Herb & - & - & + & - & - & - & - \\
\hline Gymnadenia orchidis Lindl. & Herb & - & + & - & - & - & - & - \\
\hline Herminium monorchis L. & Herb & - & - & - & + & + & - & - \\
\hline Neottia listeroides Lindl. & Herb & - & + & - & - & - & - & - \\
\hline platanthera edgeworthii (Hook.f. ex Collett) R.K.Gupta. & Herb & - & - & - & + & - & - & - \\
\hline \multicolumn{9}{|l|}{ Orobanchaceae } \\
\hline Euphrasia himalayica Wettst. & Herb & - & + & + & + & + & + & + \\
\hline Pedicularis bicornuta Klotzsch & Herb & - & - & - & - & + & + & + \\
\hline Pedicularis pectinata Benth. & Herb & - & + & + & + & - & + & + \\
\hline Pedicularis pyramidata Royle ex Benth. & Herb & + & - & + & + & + & - & + \\
\hline Pedicularis rhinanthoides Schrenk. & Herb & - & - & + & + & + & + & + \\
\hline
\end{tabular}


Singh, D. et al. / J. Appl. \& Nat. Sci. 11(1): 23 - 34 (2019)

\begin{tabular}{|c|c|c|c|c|c|c|c|c|}
\hline Papaveraceae & & & & & & & & \\
\hline Meconopsis aculeata Royle. & Herb & - & - & - & + & + & + & + \\
\hline Meconopsis bella Prain. & Herb & - & - & - & - & + & + & + \\
\hline Phytolaccaceae & & & & & & & & \\
\hline Phytolacca acinosa L. & Herb & + & - & + & + & - & - & - \\
\hline Pinaceae & & & & & & & & \\
\hline Abies pindrow Royle. & Tree & + & + & + & + & - & - & - \\
\hline Picea smithiana Wall. & Tree & + & + & + & - & - & - & - \\
\hline Pinus wallichiana A.B.Jacks. & Tree & + & + & + & - & - & - & - \\
\hline Plantaginaceae & & & & & & & & \\
\hline Digitalis lanata Ehrh. & Herb & + & - & - & - & - & - & - \\
\hline Digitalis purpurea L. & Herb & + & - & - & - & - & - & - \\
\hline Picrorhiza kurroa Royle ex Benth. & Herb & - & - & - & - & + & + & - \\
\hline Plantago himalaica Pilg. & Herb & + & + & + & - & + & + & - \\
\hline Plantago major L. & Herb & + & + & - & - & - & - & - \\
\hline Veronica laxa. L. & Herb & - & + & + & + & - & - & - \\
\hline $\begin{array}{l}\text { Wulfeniopsis amherstiana Benth. } \\
\text { Poaceae }\end{array}$ & Herb & + & + & - & - & - & - & - \\
\hline Hierochloe laxa Hook.f & Herb & - & - & - & - & + & + & - \\
\hline Polemoniaceae & & & & & & & & \\
\hline Polemonium caeruleum L. & Herb & + & - & + & + & - & - & - \\
\hline Polygonaceae & & & & & & & & \\
\hline Bistorta vivipara (L.) Delarbre subsp. vivipara. & Herb & - & - & + & + & + & + & - \\
\hline Oxyria digyna $\mathrm{L}$. & Herb & - & + & + & + & + & + & + \\
\hline Persicaria alpina (All.) H.Gross. & Herb & + & - & + & + & - & - & - \\
\hline Persicaria amplexicaulis (D.Don) Ronse Decr. & Herb & - & + & + & - & + & + & - \\
\hline Persicaria capitatum D.Don. & Herb & + & - & + & + & + & - & - \\
\hline Persicaria wallichii Wall. & Herb & + & + & + & + & - & - & - \\
\hline Polygonum affine D. Don. & Herb & - & - & + & + & + & - & + \\
\hline Polygonum vacciniifolium. L. & Herb & - & - & + & + & + & - & + \\
\hline Rheum australe D.Don Prodr. & Herb & - & - & + & + & + & - & - \\
\hline Rheum spiciforme Royle. & Herb & - & - & + & + & - & + & + \\
\hline Rumex acetosa $L$ & Herb & + & + & + & + & + & + & - \\
\hline Primulaceae & & & & & & & & \\
\hline Androsace muscoidea DC. & Herb & - & - & - & + & + & - & + \\
\hline Androsace rotundifolia Hardw. & Herb & + & + & + & + & - & - & - \\
\hline Androsace sarmentosa Wall. & Herb & + & + & + & - & - & - & - \\
\hline Primula macrophylla D. Don. & Herb & - & - & - & + & + & + & - \\
\hline Primula primulina (Spreng.) & Herb & - & - & - & - & + & & + \\
\hline Primula rosea Royle. & Herb & + & + & + & - & + & - & - \\
\hline Primula stuartii Wall. & Herb & - & - & - & + & + & - & - \\
\hline Ranunculaceae & & & & & & & & \\
\hline Aconitum heterophyllum Wall. ex Royle & Herb & + & + & - & + & + & + & - \\
\hline Aconitum leave Royle. & Herb & + & + & + & + & - & - & - \\
\hline Aconitum violaceum Jacquem. ex Stapf. & Herb & + & + & + & - & + & + & - \\
\hline Actaea spicata L. & Herb & + & + & - & + & - & - & - \\
\hline Anemone obtusiloba D.Don & Herb & + & + & + & + & + & - & - \\
\hline Anemone rupicola Cambess ex. Jacquem. & Herb & - & - & + & + & - & - & + \\
\hline Anemone tetrasepala Royle. & Herb & - & + & + & + & - & - & - \\
\hline Aquilegia pubiflora Wall. & Herb & + & + & + & - & - & - & - \\
\hline Caltha palustris $\mathrm{L}$. & Herb & - & - & + & + & + & + & - \\
\hline Clematis grata Wall. & Herb & + & - & - & - & - & - & - \\
\hline Delphinium vestitum Wall. ex Royle. & Herb & + & + & + & + & + & - & + \\
\hline Paraquilegia microphylla (Royle) J.R Drumm. & Herb & - & - & - & - & + & - & + \\
\hline Ranunculus hirtellus Royl. & Herb & - & - & - & - & + & + & + \\
\hline Ranunculus laetus Wall. ex, Hooker. & Herb & + & + & + & - & - & - & - \\
\hline Thalictrum foliolosum DC. & Herb & - & + & + & + & - & - & - \\
\hline Rosaceae & & & & & & & & \\
\hline Agrimonia pilosa Ledeb. & Herb & + & + & + & + & - & - & - \\
\hline Cotoneaster nummularius Fisch. and C.A. Mey. & Shrub & + & + & - & - & - & - & - \\
\hline Cotoneaster rotundifolius Wall. ex Lindl. & Shrub & - & + & + & + & + & - & - \\
\hline Fragaria daltoniana J.Gay. & Herb & - & + & + & - & + & - & - \\
\hline Fragaria nubicola L. & Herb & + & + & + & + & + & - & - \\
\hline & & & & & & & & $1 . .$. \\
\hline
\end{tabular}


Singh, D. et al. / J. Appl. \& Nat. Sci. 11(1): 23 - 34 (2019)

\begin{tabular}{|c|c|c|c|c|c|c|c|c|}
\hline Geum elatum Wall. ex G. Don & Herb & & + & + & + & + & + & - \\
\hline Geum roylei Wall. & Herb & + & + & + & + & - & - & - \\
\hline Geum urbanum L. & Herb & + & + & - & - & - & - & - \\
\hline $\begin{array}{l}\text { Potentilla atrosanguinea Lodd., G. Lodd. and Wild. } \\
\text { Lodd. }\end{array}$ & Herb & - & + & + & + & - & - & - \\
\hline Potentilla cuneata Wall. ex, Lehm. & Herb & - & - & + & + & - & + & + \\
\hline Potentilla nepalensis Hook. & Herb & - & - & + & + & + & + & - \\
\hline Prunus cornuta (Wall. ex Royle) Steud. & Tree & + & + & + & + & - & - & - \\
\hline Rosa macrophylla Lindl. & Shrub & + & + & + & + & + & - & - \\
\hline Rosa webbiana Lindl. & Shrub & - & + & + & & + & + & - \\
\hline Sibbaldia cuneata Hornem. ex Kuntze & Herb & - & - & + & + & + & + & + \\
\hline Sorbus cuspidata (Spach) Hedl. & Tree & - & + & + & - & - & - & - \\
\hline Sorbus microphylla Wallich. & Tree & - & - & + & + & + & + & - \\
\hline $\begin{array}{l}\text { Spiraea canescens D.Don } \\
\text { Rubiaceae }\end{array}$ & Shrub & + & + & + & - & - & - & - \\
\hline Galium aparine L. & Herb & - & + & + & + & + & - & - \\
\hline Galium asperuloides Edgew. & Herb & + & + & - & - & - & - & - \\
\hline $\begin{array}{l}\text { Himalrandia tetrasperma (Wall. ex Roxb.) } \\
\text { Salicaceae }\end{array}$ & Shrub & - & + & + & + & - & - & - \\
\hline Salix elegans Wall. & Shrub & + & + & + & + & + & + & - \\
\hline $\begin{array}{l}\text { Salix lindleyana Wall. ex Andersson. } \\
\text { Saxifragaceae }\end{array}$ & Shrub & - & - & + & + & + & + & - \\
\hline Bergenia ciliata Haworth. & Herb & - & + & + & + & + & - & + \\
\hline Bergenia stracheyi (Hook.f. and Thomson) Engl. & Herb & - & - & + & + & + & + & + \\
\hline Saxifraga moorcroftiana Wall. & Herb & - & - & - & + & + & + & + \\
\hline Saxifraga sibirica $\mathrm{L}$. & Herb & - & - & + & + & + & + & + \\
\hline $\begin{array}{l}\text { Saxifraga stenophylla Royle. } \\
\text { Scrophulariaceae }\end{array}$ & Herb & - & - & + & + & - & + & + \\
\hline Lagotis cashmeriana Rupr. & Herb & - & - & + & + & + & + & - \\
\hline Scrophularia decomposita (Royle ex Benth.) & Herb & + & + & + & - & - & - & - \\
\hline $\begin{array}{l}\text { Verbascum thapsus L. } \\
\text { Solanaceae }\end{array}$ & Herb & + & + & + & - & - & - & - \\
\hline $\begin{array}{l}\text { Atropa acuminata L. } \\
\text { Taxaceae }\end{array}$ & Herb & - & - & - & + & + & - & - \\
\hline $\begin{array}{l}\text { Taxus wallichiana Zucc. } \\
\text { Thymelaeaceae }\end{array}$ & Tree & - & - & + & - & - & - & - \\
\hline $\begin{array}{l}\text { Wikstroemia canescens Wall. ex Meisn. } \\
\text { Trilliaceae }\end{array}$ & Shrub & + & + & + & - & - & - & - \\
\hline $\begin{array}{l}\text { Trillidium govanianum Wall. ex D.Don } \\
\text { Urticaceae }\end{array}$ & Herb & - & - & + & + & - & + & - \\
\hline $\begin{array}{l}\text { Pilea umbrosa Wedd. } \\
\text { Violaceae }\end{array}$ & Herb & + & + & - & - & - & - & - \\
\hline Viola biflora L. & Herb & - & - & + & + & + & - & + \\
\hline
\end{tabular}

gymnosperms. Family Asteraceae with 37 species dominated the area followed by Rosaceae (18 species), Lamiaceae (16), Ranunculaceae (15) Orchidaceae (12) and Caryophyllaceae (8). The conifers are widely distributed especially along the lower elevations. Pinus wallichiana, Picea smithiana and Abies pindrow with interspersed patches of Taxus wallichiana dominate the ridge bases especially along the northern aspects (Sites I, II and III). Kharsu-Oak, Quercus semecarpifolia forms the climax treeline community along the ridge tops mostly facing the southern aspects (Sites III and IV). The northern aspects however harbor a mix of Betula utilis and Abies pindrow patches at lower slopes. The bare ridge tops harbor immensely rich and unique herbaceous diversity mostly comprising the threatened medicinal and aromatic plants.

Richness and Diversity: Species richness and diversity for the trees, shrubs and herbs at all the 7 sites exhibited the interesting results along the rising elevation. Maximum species richness (178 species) was recorded at Site-IV with the dominance of herbs (149 species) followed by Site-III (139 species), Site-V (121 species), Site-II (113 species), Site-I (100 species) and Site-VI (82 species). Site-VII at the highest elevation of $4000 \mathrm{~m}$ with the extreme conditions offers least survival rates and thus recorded the lowest species richness of 61 (Fig 8a) The trend in species richness followed a decreasing trend with maximum number of herbs followed by trees and shrubs at all the 7 sites (Table 2).

The values for Margalef's and Menhinick's indices have been recorded highest for the herbs (19.78, $3.53)$, shrubs $(3.91,1.38)$ and trees $(1.63,0.78)$ at sites IV, II and III, respectively. The evenness was recorded highest for the herbs (0.94), shrubs 


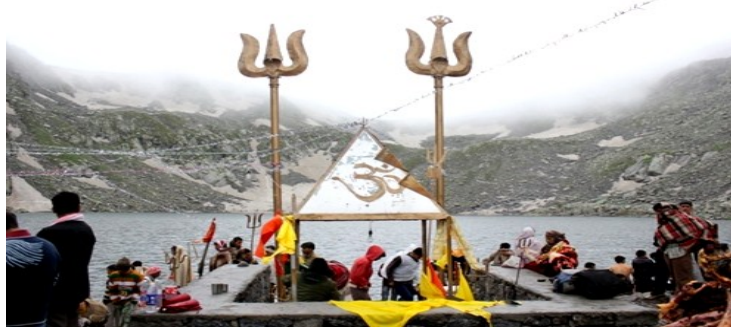

Fig. 2. Infrastructure built up at Kailash lake at $3900 \mathrm{~m}$.

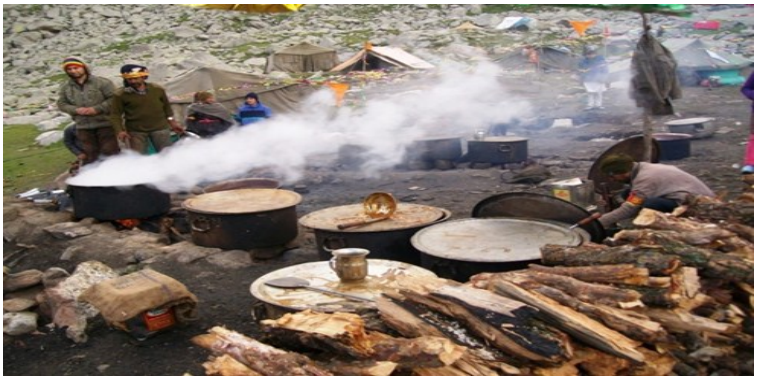

Fig. 4. Extraction of fuel wood for community kitchens.

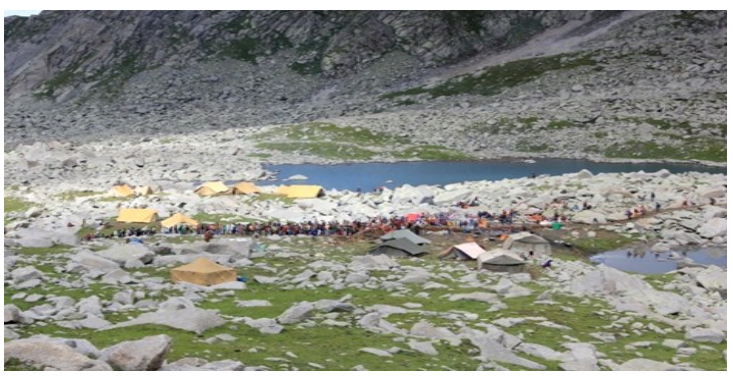

Fig. 3. Camp site for annual Kailash Yatra at $3900 \mathrm{~m}$.

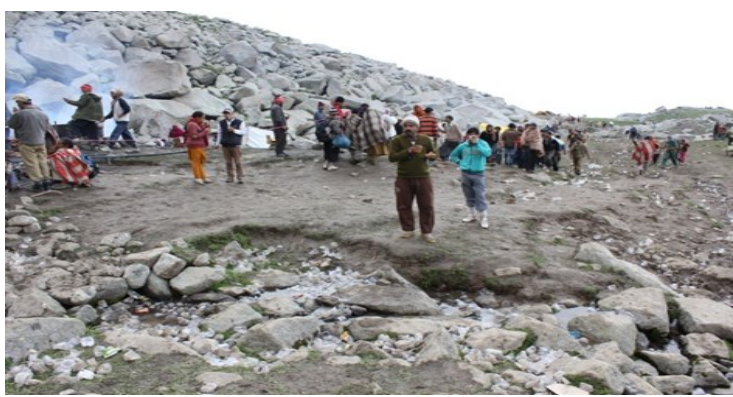

Fig. 5. Plastic pollution / trampling of ground flora .

Table 1. Floristic pattern along the elevational gradient in the study area.

\begin{tabular}{|c|c|c|c|c|c|c|c|c|c|c|c|c|c|c|c|c|c|c|c|c|c|c|}
\hline \multirow[t]{2}{*}{$\begin{array}{l}\text { Attrib- } \\
\text { utes }\end{array}$} & \multirow{2}{*}{$\begin{array}{l}\text { Whole } \\
\text { study } \\
\text { area }\end{array}$} & \multicolumn{3}{|c|}{$\begin{array}{c}\text { Site I } \\
(2800 \mathrm{~m})\end{array}$} & \multicolumn{3}{|c|}{$\begin{array}{c}\text { Site II } \\
(3000 \mathrm{~m})\end{array}$} & \multicolumn{3}{|c|}{$\begin{array}{c}\text { Site III } \\
(3200 \mathrm{~m})\end{array}$} & \multicolumn{3}{|c|}{$\begin{array}{c}\text { Site IV } \\
(3400 \mathrm{~m})\end{array}$} & \multicolumn{3}{|c|}{$\begin{array}{c}\text { Site V } \\
(3600 \mathrm{~m})\end{array}$} & \multicolumn{3}{|c|}{$\begin{array}{c}\text { Site VI } \\
(3800 \mathrm{~m})\end{array}$} & \multicolumn{3}{|c|}{$\begin{array}{c}\text { Site VII } \\
(4000 \mathrm{~m})\end{array}$} \\
\hline & & $T$ & $S$ & H & $T$ & $S$ & H & $T$ & $\mathrm{~s}$ & H & $T$ & $\mathrm{~s}$ & H & $T$ & S & H & $\mathbf{T}$ & S & $\mathrm{H}$ & $T$ & S & $\mathrm{H}$ \\
\hline Species & 253 & 8 & 14 & 78 & 8 & 23 & 82 & 9 & 20 & 110 & 9 & 20 & 149 & 1 & 14 & 106 & 0 & 6 & 76 & 0 & 2 & 59 \\
\hline Genera & 170 & 8 & 13 & 63 & 8 & 18 & 66 & 9 & 16 & 82 & 8 & 15 & 98 & 1 & 10 & 78 & 0 & 4 & 59 & 0 & 2 & 49 \\
\hline $\begin{array}{l}\text { Fami- } \\
\text { lies }\end{array}$ & 62 & 5 & 10 & 30 & 6 & 15 & 32 & 6 & 14 & 34 & 6 & 12 & 38 & 1 & 7 & 32 & 0 & 2 & 23 & 0 & 1 & 21 \\
\hline
\end{tabular}

Where, T - Trees, S - Shrubs and H- Herbs

Table 2. Species richness along the rising elevation.

\begin{tabular}{lllllllll}
\hline Life form & \multicolumn{9}{c}{ Study sites } & \multicolumn{2}{c}{\begin{tabular}{l} 
Whole study area \\
\cline { 2 - 8 }
\end{tabular}} & I & II & III & IV & V & VI & VII & (nos) \\
\hline Trees & 8 & 8 & 9 & 9 & 1 & 0 & 0 & 11 \\
Shrubs & 14 & 23 & 20 & 20 & 14 & 6 & 2 & 29 \\
Herbs & 78 & 82 & 110 & 149 & 106 & 76 & 59 & 213 \\
Total & $\mathbf{1 0 0}$ & $\mathbf{1 1 3}$ & $\mathbf{1 3 9}$ & $\mathbf{1 7 8}$ & $\mathbf{1 2 1}$ & $\mathbf{8 2}$ & $\mathbf{6 1}$ & $\mathbf{2 5 3}$ \\
\hline
\end{tabular}

Table 3. Plant species richness, diversity, dominance and evenness along the rising elevation in the study area.

\begin{tabular}{lllllllll}
\hline Parameter & Taxa & Site I & Site II & Site III & Site IV & Site V & Site VI & Site VII \\
\hline Species rich- & Trees & 8 & 8 & 9 & 9 & 1 & 0 & 0 \\
ness & Shrubs & 14 & 23 & 20 & 20 & 14 & 6 & 2 \\
& Herbs & 78 & 82 & 110 & 149 & 106 & 76 & 59 \\
Total & & 100 & 113 & 139 & 178 & 121 & 82 & 61 \\
Shanon Wie- & Trees & 1.533 & 1.494 & 1.745 & 1.029 & 0 & 0 & 0 \\
ner's Index & Shrubs & 2.308 & 2.452 & 2.485 & 2.419 & 2.307 & 1.234 & 0.4101 \\
& Herbs & 3.966 & 3.945 & 4.27 & 4.604 & 4.355 & 4.086 & 3.805 \\
Pielou's Even- & Trees & 0.7370 & 0.7184 & 0.7942 & 0.4681 & 0 & 0 & 0 \\
ness Index & Shrubs & 0.8744 & 0.7819 & 0.8295 & 0.8074 & 0.8741 & 0.6889 & 0.5917 \\
& Herbs & 0.9102 & 0.8953 & 0.9084 & 0.9201 & 0.9338 & 0.9436 & 0.9333 \\
Simpson's & Trees & 0.7215 & 0.7055 & 0.7983 & 0.4566 & 0 & 0 & 0 \\
Index & Shrubs & 0.8748 & 0.8581 & 0.8911 & 0.8775 & 0.8779 & 0.6167 & 0.2449 \\
& Herbs & 0.9697 & 0.9725 & 0.9806 & 0.9863 & 0.9844 & 0.9801 & 0.9718 \\
Menhinick's & Trees & 0.7493 & 0.7396 & 0.7804 & 0.7324 & 0.5 & 0 & 0 \\
Index & Shrubs & 1.043 & 1.387 & 1.339 & 0.9975 & 0.6585 & 0.5324 & 0.252 \\
& Herbs & 3.305 & 2.636 & 2.994 & 3.537 & 2.915 & 2.79 & 2.995 \\
Margalef's & Trees & 1.478 & 1.47 & 1.636 & 1.594 & 0 & 0 & 0 \\
index & Shrubs & 2.503 & 3.917 & 3.514 & 3.169 & 2.126 & 1.032 & 0.2414 \\
& Herbs & 12.18 & 11.78 & 15.12 & 19.78 & 14.61 & 11.35 & 9.73 \\
\hline \hline
\end{tabular}




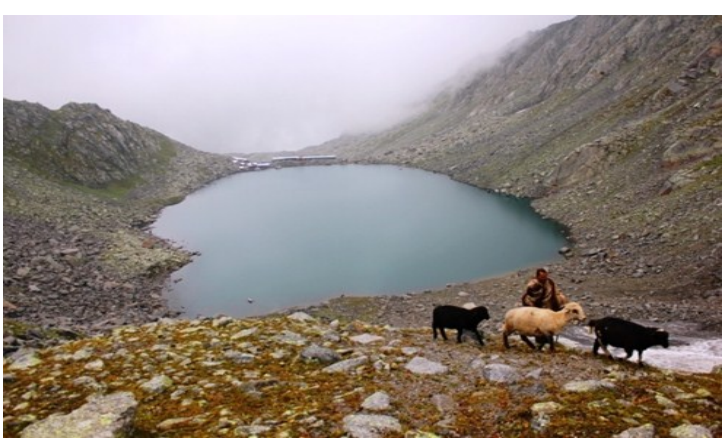

Fig. 6. Live stock grazing around Kaialsh Lake .

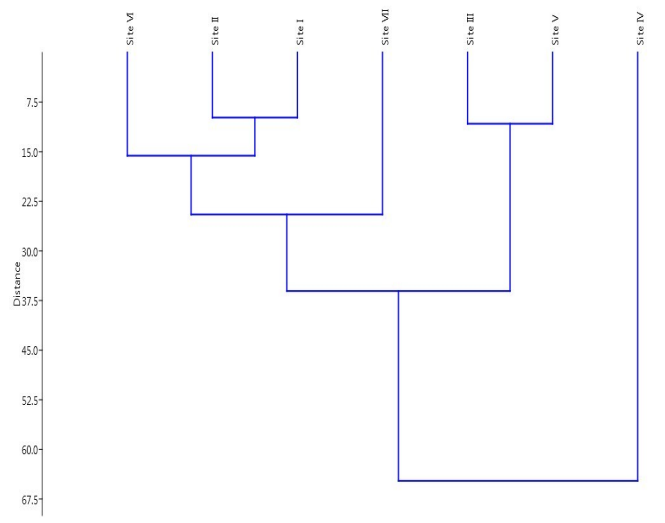

Fig. 8. Hierarchal cluster analysis a) Species richness b) Species diversity.

(0.87) and trees $(0.79)$ at sites $\mathrm{VI}, \mathrm{I}$ and III, respectively. The species diversity was recorded maximum for herbs $\left(H^{\prime}=4.60\right)$ at site IV, shrubs $\left(H^{\prime}=2.48\right)$ at site III and trees $\left(H^{\prime}=1.74\right)$ at site III, respectively (Fig $8 b)$. The value of Simpson's index was recorded maximum for herbs $(1 D=0.98)$ at Site IV, for shrubs (0.89) and trees (0.79) at site III (Table 3).

\section{DISCUSSION}

The study area is represented by sub-alpine to alpine vegetation with broadleaved-conifer mixed stands at lower elevations taken over by KharsuOak climax community and alpine scrub along the rising elevation from $2800 \mathrm{~m}$ to $4000 \mathrm{~m}$ in upper Bhaderwah catchment. During their floristic analysis in Bhaderwah and vicinity, Kumar (1987), Kumar (1997), Singh and Rawat (2000), Kour (2001), Dutt (2005), Raina and Kumar (2011), Sharma et al. (2016) and Sharma (2018) reported similar trend in species composition, richness, and dominance with a characteristic mid-domain effect exhibited by Sites III, IV and V (Fig 8a). Our observation is in consonance with the results obtained from similar landscapes (Singh and Rawat, 2000; Kessler, 2000; Grytnes and Vetaas, 2002; Chawla et al., 2008 and Habib et al., 2011). The

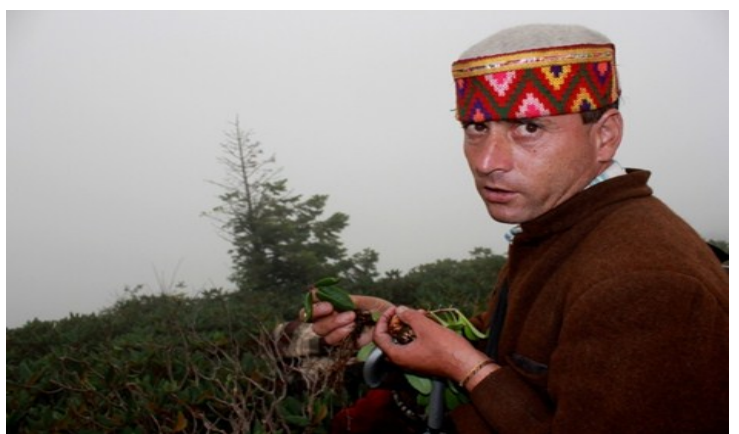

Fig. 7. Extraction of medicinal plants.

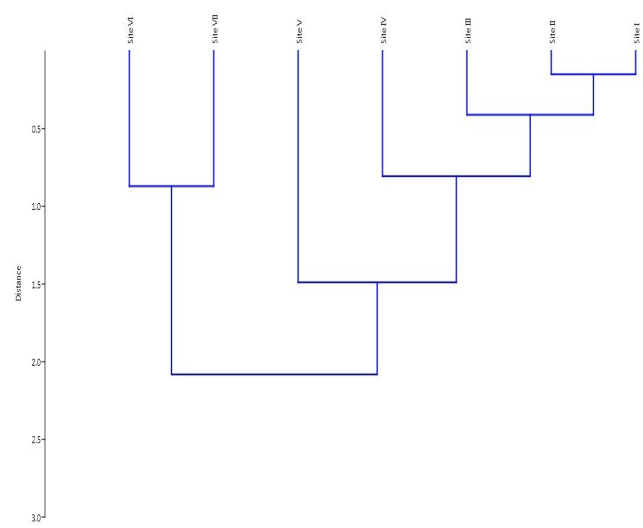

herbs contribute maximum in terms of species richness and diversity recorded highest at midelevations (Site IV) with a corresponding decline along the rising elevation (Kharkwal et al., 2005). A sharp dip in species richness is observed beyond Site IV with a minima recorded at Site VII (62 species). The reduction in species at higher altitudinal gradient could be due to the effect of ecophysiological constraints, such as short period of growing season, extremely low temperature, and geographical barriers (Grytnes and Beaman, 2006).

In terms of species diversity, various indices (Shannon-Wiener's, Margalef's, Menhinick's and Simpson's index) were calculated for all the layers i.e., trees, shrubs and herbs. . The richness and diversity values showed an incremental rise from hill base (Site I) till mid-altitude (Site IV) with a gradual fall corresponding to the rising elevation further. The higher diversity values in terms of Margalef's Index (1.63 for trees / 19.78 for herbs), Menhinick's Index (0.78 / 3.53) were observed at sites III and IV, respectively. Shrubs on the other hand showed a bit different trend where the richness and diversity were recorded maximum at lower elevation (Margalef's Index $=3.91$ / Menhinick's Index $=1.38$ ) at Site II with a slight dip in 
species richness with the rising elevation. The dendrogram for diversity (Fig 8b) clearly explains this trend. This finding is in agreement with the studies by Kumar and Ram (2005), Kharkwal et al. (2005), Sharma et al. (2009), Bargali et al. (2013) and Sharma and Raina (2013). In terms of trees, Quercus semecarpifolia singly dominates Site IV while Site VII indicated the prevalance of shrubs. The herbs dominated all the seven sites. The reason for dominance of single or few species at these sites may be due to sudden changes in the extreme weather conditions in this zone resulting in the occurrence of only the tolerant species (Singh and Rawat, 2000). The occurrence of Quercus semecarpifolia and Betula utilis along the sub-alpine altitudinal gradient suggests their tolerance to biotic pressures and wider ecological amplitude wherein Kharsu-Oak is a climatic climax (Champion and Seth, 1968).

\section{Conclusion}

The investigations revealed that mid-elevations support higher species richness and diversity than the cold and higher elevational cover types, which implies that sub-alpine and alpine forests need effective monitoring and conservation. The study suggests that the distribution and species richness pattern of different tree species are largely regulated by altitude and climatic factors.

\section{ACKNOWLEDGEMENTS}

Authors gratefully acknowledge Rector Bhaderwah campus for providing the financial assistance to carry out the field surveys. The Department of Forests and Wildlife Protection, Govt. of Jammu and Kashmir is duly acknowledged for their help and support during the investigations.

\section{REFERENCES}

1. Bargali, K., Bisht, P., Khan, and Rawat, Y. S. (2013). Diversity and regeneration status of tree species at Nainital catchment, Uttarakhand. India. International journal of biodiversity and conservation, 5(5): 270280.

2. Butchart, S.H.M., Walpole, M., Collen, B., van Strien, A., Scharlemann, J.P.W., Almond, R.E.A. (2010) Global Biodiversity: Indicators of Recent Declines. Science. 2010; 328(5982):1164-8. https:// doi.org/10.1126/ science.1187512 PMID: 20430971

3. Cairns, D. M. (1998). Modeling controls on pattern at alpine treeline. Geographical and Environmental Modeling, 2(1):43-63.

4. Cairns, D.M. and Malanson G.P. (1997). Examination of the Carbon Balance Hypothesis of Alpine Treeline Location in Glacier National Park, Montana. Physical Geography, 18(2): 125-145.

5. Cairns, D.M. and Malanson G.P. (1998). Environmental variables influencing the carbon balance at the alpine treeline: a modeling approach. Journal of vegetation science, 9(5): 679-692.

6. Champion, H. G. and S. K. Seth (1968). A revised survey of the forest types of India. Manager of Publi- cations, Govt. of India, New Delhi.

7. Chapin III, Erika, F.S., Zavaleta, S., Eviner, V. T., Naylor, R. L., Vitousek, P. M., Reynolds, H. L., Hooper, D. U., Lavorel, S., Sala, O. E., Hobbie, S. E., Mack, M. C. And Diaz S. (2000). Consequences of changing biodiversity. Nature, 405: 234-242.

8. Chawla, A., Rajkumar S., Singh, K. N., Brij. L. and Sigh, R. D. (2008). Plant species Diversity along Altitudinal Gradient of Bhabha Valley in Western Himalayas. Journal of Mountain. Science, 5: 157-177.

9. Dutt, H. (2005). Ecological studies and conservation of medicinal plants of Neeru watershed ( $J$ and $K$ ). $\mathrm{Ph} . \mathrm{D}$ thesis. University of Jammu, Jammu, India.

10.Elourard, C., Pascal, J. P., Pelissier, R., Ramesh, B. R., Houllier, F., Durand, M, Aravajy, S, Moravie, M. A., Gimaret-Carpentier, C. (1997). Monitoring the structure and dynamics of a dense moist evergreen forest in the Western Ghats (Kodagu District, Karnataka, India). Tropical Ecolog, 38: 193-214.

11.Grytnes, J, A. and Beaman, J. H. (2006). Elevational species richness pattern for vascular plants on Mount Kinabalu, Borneo. Journal of biogeography, 33: 18381849.

12.Grytnes, J. A. and Vetaas, O. R. (2002). Species richness and altitude: A comparison between null models and interpolated plant species richness along the Himalayan altitudinal gradient, Nepal. American Naturalist, 159: 294-304.

13. Habib, T., Malik, Z., Hussain, M. and Khan, M. (2011). Plant species diversity along the altitudinal gradient at Garhi Dopatta Hills, Muzaffarabad. Journal of Medicinal Plants Research, 5(20): 5194-5196.

14. Hall, H. P. and Daniel B. Fagre (2003). Modeled climate-induced glacier change in Glacier National Park, 1850-2100. Bioscience, 53(2) 131-140.

15. Holtmeier, F. K. (2003). Mountain Timberlines: Ecology, Patchiness, and Dynamics, Kluwer Academic Publishers, Netherlands: 384

16. Holtmeier, F.-K. and Broll, G. (1992). The Influence of Tree Islands and Microtopography on Pedogeological Conditions in the Forest-Alpine Tundra Ecotone on Niwot Ridge, Colorado Front Range, U.S.A. Arctic and Alpine Research, 24(3): 216-228.

17.Kosanic ,A., Anderson, K., Harrison, S., Turkington, T., Bennie, J (2018). Changes in the geographical distribution of plant species and climatic variables on the West Cornwall peninsula (South West UK). PLOS ONE 13(2): e0191021. https://doi.org/10.1371/ journal.pone. 0191021

18.Kessler, M. (2000). Elevational gradients in species richness and endemism of selected plant groups in the central Bo-Livian Andes. Plant Ecology, 149: 181193.

19.Kharkwal, G., Mehrotra, P., Rawat, Y. S. and Pangtey, Y. P. S. (2005). Phytodiversity of growth form in relation to altitudinal gradient in the Central Himalayan (Kumaun) region of India. Current Science, 89 (5): 873-878.

20.Kour, I. (2001). Phytodiversity and impact of tourism on the vegetation of Trikuta Hills ( $J$ and K). Ph.D Thesis. University of Jammu, Jammu, India.

21.Kumar A, Ram J. (2005). Anthropogenic disturbances and plant biodiversity in forests of Uttaranchal, Central Himalayas. Biodiversity Conservation, 14: 309-331.

22.Kumar, A. (1987). Phytosociological and productivity studies of Bhaderwah forests, Jammu ( $J$ and $K$ ). 
Ph.D Thesis. University of Jammu, Jammu, India.

23.Kumar, K. (1997). Studies on plant diversity of Patnitop and adjoining area and impact of biotic activities. Ph.D Thesis. University of Jammu, Jammu, India.

24.Margalef, R. (1968). Perspectives in ecological theory. University of Chicago Press. pp 111.

25.Menhinick, E. F. (1964). A comparison of some Species Diversity Indices applied to Samples of Field Insects. Ecology, 45: 858-862.

26.Pacifici, M., Foden, W.B., Visconti, .P, Watson, J.E.M., Butchart, S.H.M., Kovacs, K.M (2015). Assessing species vulnerability to climate change. Nature Climate Change. 5(3):215-24. https:// doi.org/10.1038/ nclimate2448

27.Parmesan, C., Duarte, C., Poloczanska, E., Richardson, A.J., Singer, M.C (2011) Overstretching attribution. Nature Climate Change. 1(1):2-4.

28.Pielou, E. C. (1966). The measurement of diversity in different types of biological collections. Journal Theoretical Biology, 13:131-144.

29.Polunin, O. and Stainton, A. (1984). Flowers of Himalaya. Oxford University Press New Delhi. 580.

30.Raina, A. K., Kumar, R. (2011). Floristic composition, life form classification and biological spectrum of the Biological spectrum of the catchment of Rattle H.E. project, District Kishtwar- JandK. Environ. Conserv. J., 12(3): 1-6.

31.Richardson, A.D., Andy Black. T., Ciais, P., Delbart, N., Friedl, M.A., Gobron, N. (2010) Influence of spring and autumn phenological transitions on forest ecosystem productivity. Philosophical Transactions of the Royal Society of London B: Biological Sciences, 365(1555):3227-46. https://doi.org/10.1098/ rstb.2010.0102 PMID: 20819815

32.Rochefort, R., Little, R.L., Woodward, A. and Peterson, D. L. (1994). Changes in sub-alpine tree distribution in western North America: A review of climatic and other causal factors. The Holocene 4(1): 89-100.
33.Shannon, C. E. and Wener, W. (1963). The Mathematical Theory of Communication, University of Illinois Press, Urbana, 117

34.Sharma, A. (2018). Vegetation composition, pattern and diversity of riparian forest communities along Neeru stream, Bhaderwah, JandK. Ph.D Thesis. University of Jammu, Jammu, India.

35.Sharma, C. M., Suyal, S., Gairola, S., and Ghildiyal, S. K. (2009). Species richness and diversity along an altitudinal gradient in moist temperate forest of Garhwal Himalaya. Journal of American Science, 5(5): 119-128.

36.Sharma, N. and Raina, A. K. (2013). Composition, structure and diversity of tree species along an elevational gradient in Jammu province of north-western Himalayas, Jammu and Kashmir, India. Journal of Biodiversity and Environmental Sciences, 3(10):12-23

37.Sharma, N., Najeeb, A. and Singh, D. (2016). Vegetational diversity and distribution along an elevational gradient on both sides of Chattergalla ridge, Bhaderwah, Jammu and Kashmir, India. Indian Forester. 142(9): 820-826.

38.Simpson, E. H. (1949). Measurement of diversity. Nature, 163: 688.

39.Singh, S. K. and Rawat, G. S. (2000). Flora of Great Himalayan National Park. Bishen Singh Mahendra Pal Singh, Dehradun, $304 \mathrm{p}$.

40.Sykes, M. T. (2009) Climate Change Impacts: Vegetation. In: Encyclopedia of Life Sciences (ELS). John Wiley and Sons, Ltd: Chichester. DOI: 10.1002/9780470015902.a0021227

41.Vila, M. and Ibanez,I. (2011). Plant invasions in the landscape. Landscape Ecol. 26(4):461-72. https:// doi.org/ 10.1007/s10980-011-9585-3

42.Wardle, P. (1974). Alpine timberlines. In: Ives, J. D. and R. G. Barry (Ed) Arctic and Alpine Environment, Methuen, London: pp371-402

43.Whittaker R. H. (1972). Evolution and Measurement of Species Diversity. Taxon, 21(2/3): 213-251. 